\title{
Optimization of AMI-MDM-RoFSO under atmospheric turbulence
}

\author{
Sushank Chaudhary ${ }^{1}$ and Angela Amphawan ${ }^{1,2}$ \\ ${ }^{1}$ Optical Computing and Research Laboratory, School of Computing, Universiti Utara Malaysia, 06010 Sintok, Kedah, Malaysia \\ ${ }^{2}$ Research Laboratory of Electronics, Massachusetts Institute of Technology, 02139 Cambridge, Massachusetts, USA
}

\begin{abstract}
Radio over Free Space (Ro-FSO) is promising candidate for providing ubiquitous digital services especially in rural areas. This work investigates the performance of MDM of two 5Gbps-10 GHz data channels over FSO link using LP 01 and LP 02 modes under the effect of atmospheric turbulences. The signal to noise ratio (SNR), total received power, modal decomposition at receiver at the receiver is also reported. The reported result shows the successful transmission of two channels with acceptable SNR over FSO link under atmospheric turbulences.
\end{abstract}

\section{Introduction}

The plethora of wireless network platforms includes numerous operators with independent radio base stations and networks. The diversity of wireless services leads to excessive investment on infrastructure and redundancy of equipment, which may affect cellular architecture efficiency. According to reports by the International Telecommunication Union (ITU), cellular services witnessed a remarkable growth of 7.5 billion subscribers in 2013 [1]. Despite this, radio frequency is spectrum is still a scarce commodity.

The limitation of radio frequency amidst the growth in mobile subscribers has motivated the development of Ro-FSO, a technology that transmits multiple RF signals through a high-speed optical carrier without investing excessively on optical fiber cabling [2, 3]. As Ro-FSO utilizes optical carriers to exploit various electromagnetic spectrum for mobile networks, it efficiently eliminates congestion issues and licensing of RF signals prevalent in the current wireless networks. Ro-FSO is a multi-faceted technology that combines radio-over-fiber (RoF) and free-space optical (FSO) technologies. RoF is used for centralizing processes including RF handoff, up-down conversion, coding, switching, and multiplexing among the base stations [4]. FSO is used for transporting data signals at a higher bandwidth through atmosphere than radio signals. Thus, Ro-FSO is efficient for integrating radio and optical networks [5].

However, the performance of Ro-FSO can be affected by atmospheric turbulences arising from fog, rain and snow [6]. In order to achieve high capacity in RoFSO while optimizing network resources, researchers have highlighted the use of advanced modulation formats [7]. Alternate mark inversion (AMI) has been used for improving the capacity of fiber transmission. AMI is a bipolar encoding system which has binary 0 as the neutral voltage and binary 1 as the alternating positive and negative voltages. However, AMI has not been exploited in free-space optical network systems. Another bandwidth-enhancing strategy, known as mode division multiplexing (MDM), is used for enhancing the capacity of free-space optical networks [8-12] by leveraging the abundance of fiber eigenmodes for transmitting various channels by generating specific modes by means of optical signal processing [13-15], spatial light encoding [16-18], few mode fibers [19].

The current study aims to utilize both aforementioned strategies, specifically a new combination of AMI and MDM in Ro-FSO systems for increasing the capacity and transmission distance, which thus far has not been covered in any previous studies. In order to achieve this objective, a simulation of an MDM system on Linearly Polarized (LP) 01 and 02 modes using AMI-encoded signals will be performed over a $3 \mathrm{~km}$ FSO link to optimize the parameters of the system under various atmospheric conditions.

\section{System Description}

MDM-RoFSO modelled in OptiSystem ${ }^{\mathrm{TM}}$. AMI is used for $5 \mathrm{Gbps}$ data encoding while its line codes are

\footnotetext{
* Corresponding author: sushankchaudhary@gmail.com
} 
generated using delay-and-add and delay-and-subtract operations $[20,21]$. Data is transmitted through pulse amplitudes of on-off-keying (OOK) while impressing its phase modulation using mathematical operations of binary input signal $\mathrm{x}(\mathrm{n})=[22]$ and output signal yАMI $(\mathrm{n})$ as:

$y_{A M I}(n)=x(n)-x(n-1)$

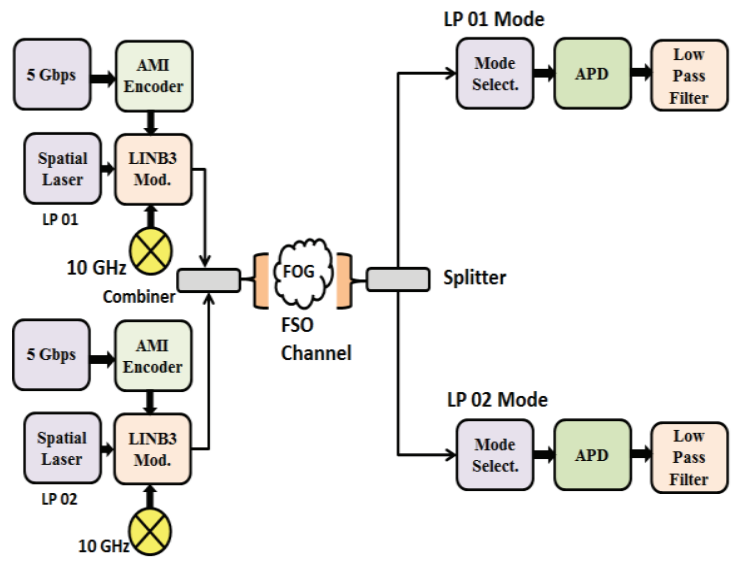

Fig. 1. Proposed Ro-FSO system

The two channels are launched from LP 01 mode and LP 02 mode, where $\mathrm{y}_{\mathrm{AMI}}$ is the AMI sequence, modulated using a Lithium Niobate dual-port optical modulator and $10 \mathrm{GHz}$ radio signal through a $850 \mathrm{~nm}$ spatial laser respectively after propagation through a multimode fiber.

The two modes are transmitted over a $3 \mathrm{~km}$ FSO along with the pre and post compensation using optical amplifiers. The FSO link equation is given as [24]:

$$
P_{\text {Received }}=P_{\text {Transmitted }} \frac{d_{R}^{2}}{\left(d_{T}+\theta_{R}\right)^{2}} 10^{\alpha R / 10}
$$

where $d_{R}$ is the receiver aperture diameter, $d_{T}$ is the transmitter aperture diameter, $\theta$ is the beam divergence, $R$ the range, and $\alpha$ the atmospheric attenuation.

The performance of the proposed Ro-FSO system under specified atmospheric conditions is investigated by adjusting the atmospheric attenuation value for different atmospheric conditions, i.e, $0.14 \mathrm{~dB}$ for clear weather conditions, $4 \mathrm{~dB}$ for hazy weather, $9 \mathrm{~dB} / \mathrm{km}$ for thin fog, $13 \mathrm{~dB} / \mathrm{km}$ for light fog, $16 \mathrm{~dB} / \mathrm{km}$ for thick fog, and 22 $\mathrm{dB} / \mathrm{km}$ for heavy fog $[25,26]$.

An Avalanche photodetector with low pass filter is used for recovering the original baseband transmitted signal. The mean squared error minimization of the estimate of sum of electric fields and the output field is performed [27] to retrieve the modal composition at the receiver [27].

\section{Results and Discussion}

As shown in Fig. 2, the signal to noise ratio (SNR) at the receiver side for Channel 1 and Channel 2 is measured with respect to FSO range under various atmospheric turbulences. The following SNR values are calculated for channel 1 under different atmospheric conditions: 71.11 $\mathrm{dB}, 54.32 \mathrm{~dB}$, and $22.31 \mathrm{~dB}$ for hazy weather; $68.21 \mathrm{~dB}$, $38.32 \mathrm{~dB}$, and $0 \mathrm{~dB}$ for light fog; $67.34 \mathrm{~dB}, 27.41 \mathrm{~dB}$, and $0 \mathrm{~dB}$ for moderate fog; and $66.23 \mathrm{~dB}, 18.43 \mathrm{~dB}$, and $0 \mathrm{~dB}$ for heavy fog at the FSO range of $500 \mathrm{~m}, 1625 \mathrm{~m}$, and $5000 \mathrm{~m}$ respectively.

Fig. 3 shows the following values of the total power calculated at the receiver side with respect to FSO range for channel 1 under various atmospheric conditions: $17.35 \mathrm{dBm},-45.10 \mathrm{dBm}$, and $-91.67 \mathrm{dBm}$ for hazy weather; $-22.11 \mathrm{dBm},-61.78 \mathrm{dBm}$, and $-100 \mathrm{dBm}$ for light fog; $-25.10 \mathrm{dBm},-71.28 \mathrm{dBm}$, and $-100 \mathrm{dBm}$ for moderate fog; and $-28.05 \mathrm{dbm},-81.36 \mathrm{dBm}$, and $-100 \mathrm{dBm}$ for heavy fog at the FSO range of $500 \mathrm{~m}, 1625 \mathrm{~m}$, and $5000 \mathrm{~m}$ respectively.

From Fig. 2 and Fig. 3, it is evident that the FSO link reach is extended under various atmospheric conditions: $3900 \mathrm{~m}$ in hazy weather, $2700 \mathrm{~m}$ in light fog, $2100 \mathrm{~m}$ in moderate fog, and $1600 \mathrm{~m}$ in heavy fog with acceptable SNR values for channel 1 . The largest amount of power for channel 1 is coupled into LP 01 mode, as shown in Fig. 4.

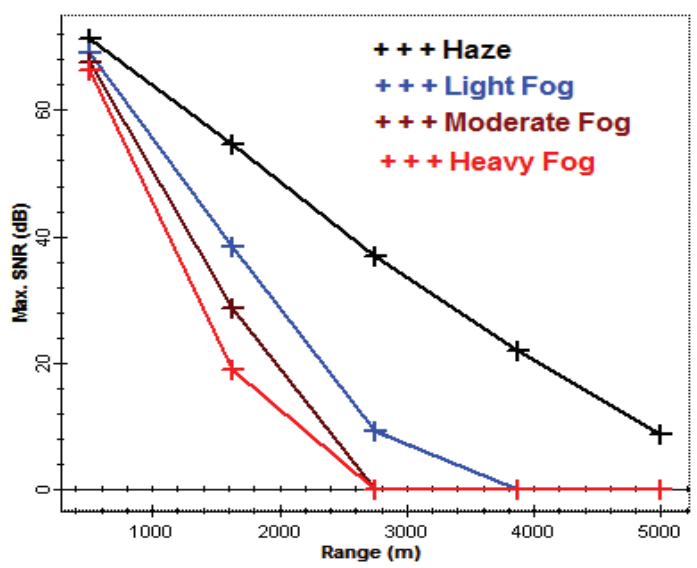

Fig. 2. SNR versus range under different weather conditions (Channel 1)

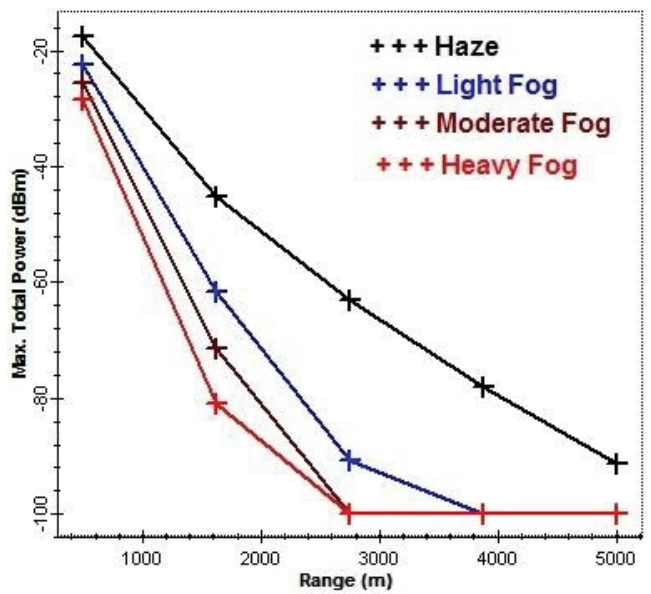

Fig. 3. Total received power versus range (Channel 1) 


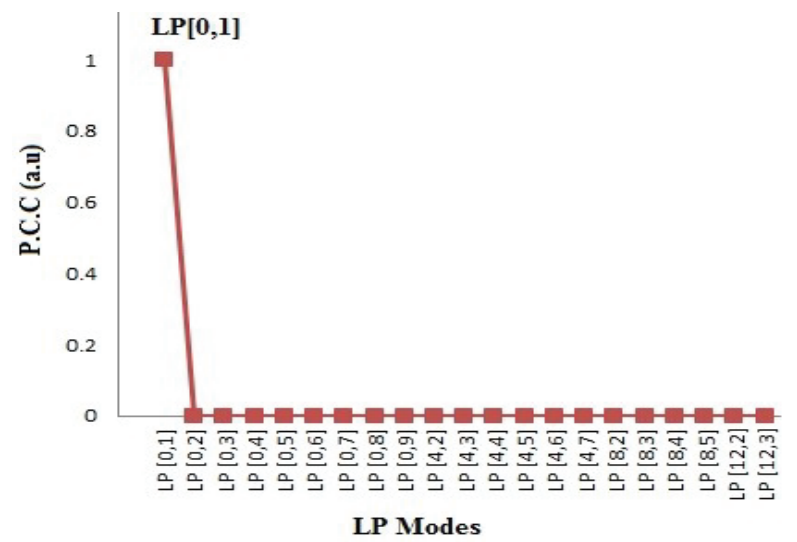

Fig. 4 Power coupling coefficients in terms of LP Modes for Channel 1

\section{SNR versus FSO Range (Channel 2)}

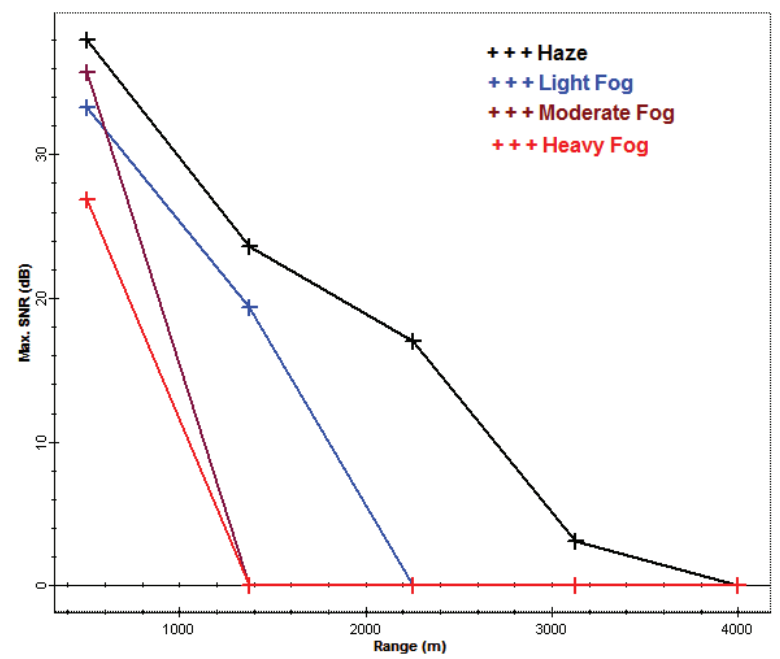

Fig. 5 SNR versus range under different weather conditions (Channel 1)

\section{Total Received Power versus FSO range Channel 2}

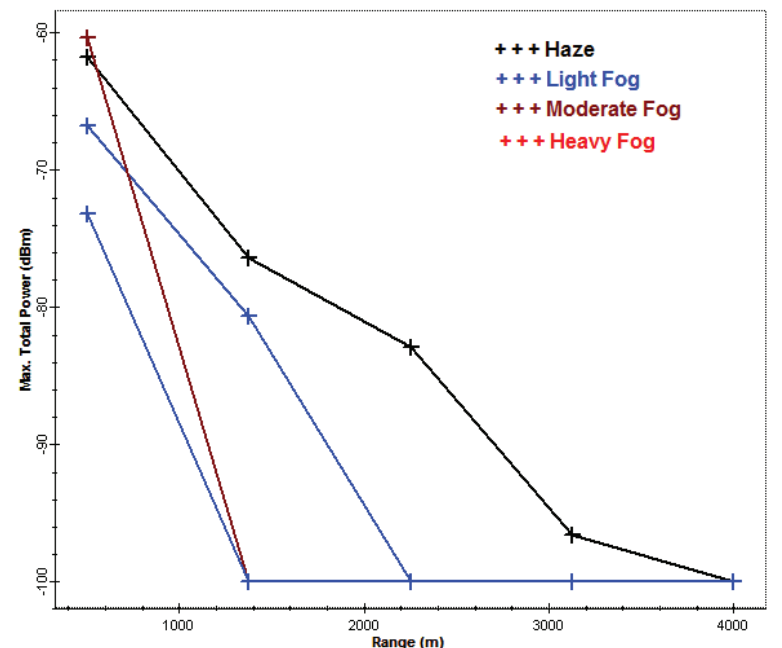

Fig. 6 Total received power versus range (Channel 2)

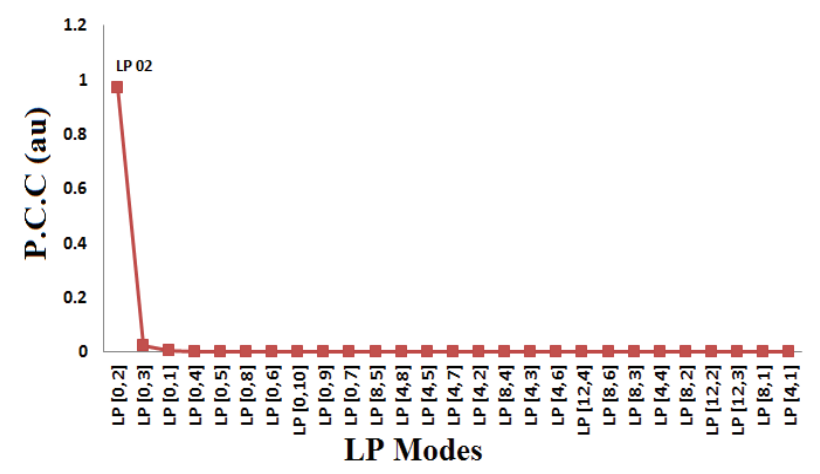

Fig 7 Power coupling coefficients in terms of LP Modes for Channel 2

Similarly, the following SNR values are calculated for channel 2 under different atmospheric conditions: 37.91 $\mathrm{dB}, 17.23 \mathrm{~dB}$, and $0 \mathrm{~dB}$ for hazy weather; $33.87 \mathrm{~dB}, 0 \mathrm{~dB}$, and $0 \mathrm{~dB}$ for light fog; $35.68 \mathrm{~dB}, 0 \mathrm{~dB}$, and $0 \mathrm{~dB}$ for moderate fog; and $26.81 \mathrm{~dB}, 0 \mathrm{~dB}$, and $0 \mathrm{~dB}$ for heavy fog at the FSO range of $500 \mathrm{~m}, 2250 \mathrm{~m}$, and $4000 \mathrm{~m}$ respectively as shown in Fig. 5. Consequently, Fig. 7 shows the following values of the total power calculated at the receiver side with respect to FSO range for channel 1 under various atmospheric conditions: $-61.23 \mathrm{dBm}$, $82.45 \mathrm{dBm}$, and $-100 \mathrm{dBm}$ for hazy weather; $-66.45 \mathrm{dBm}$, $-100 \mathrm{dBm}$, and $-100 \mathrm{dBm}$ for light fog; $-60.23 \mathrm{dBm},-100$ $\mathrm{dBm}$, and $-100 \mathrm{dBm}$ for moderate fog; and $-73.10 \mathrm{dbm}$, $100 \mathrm{dBm}$, and $-100 \mathrm{dBm}$ for heavy fog at the FSO range of $500 \mathrm{~m}, 2250 \mathrm{~m}$, and $4000 \mathrm{~m}$ respectively.

From Fig. 5 and Fig. 6, it is evident that the FSO link reach for channel 2 is extended under various atmospheric conditions: $2200 \mathrm{~m}$ in hazy weather, $1375 \mathrm{~m}$ in light fog, $1050 \mathrm{~m}$ in moderate fog, and $900 \mathrm{~m}$ in heavy fog with acceptable SNR values. The largest amount of power for channel 1 is coupled into LP 02 mode, as shown in Fig. 7.

\section{Conclusion}

Numerical simulations demonstrate that MDM system on LP 01 mode and LP 02 mode using AMI-encoded signals are viable for extending the range of the FSO link with acceptable SNR. The results show that the for channel 1, FSO links prolong to $3.9 \mathrm{~km}$ in hazy weather whereas the links prolong to $2.7 \mathrm{~km}$ in case of light fog. In case of moderate fog, the FSO links prolong to $2.1 \mathrm{~km}$ and to 1.6 $\mathrm{km}$ in heavy fog. For channel 2, FSO link prolongs to $2200 \mathrm{~m}$ in hazy weather whereas $1375 \mathrm{~m}$ in light fog. In case of moderate fog, FSO link prolongs to $1050 \mathrm{~m}$ and to $900 \mathrm{~m}$ in heavy fog.

\section{References}

1. I. Data, "Statistics Division Telecommunication Development Bureau ITU,"The world in 2013: Ict facts and figures," ITU Int. Telecom. Union (2013) 
2. A. Amphawan, S. Chaudhary, and V. Chan, Journal of the European Optical Society-Rapid publications, 9 (2014)

3. K. Kazaura, K. Wakamori, M. Matsumoto, T. Higashino, K. Tsukamoto, and S. Komaki, Communications Magazine, IEEE, 48, no. 2, pp. 130-137 (2010)

4. V. Thomas, M. El-Hajjar, and L. Hanzo, Performance improvement and cost reduction techniques for radio over fiber communications (2015)

5. Angela Amphawan, S. Chaudhary, R. Din, and M. N. Omar, IEEE International Colloquium on Signal Processing and its Applications (CSPA), 6-9th March 2015, Kuala Lumpur (2015)

6. S. Chaudhary and A. Amphawan, "Journal of Optical Communications, 35, no. 4, pp. 327-334 (2014)

7. K. Mishina et al., Optics Express, vol. 15, no. 12, pp. 7774-7785 (2007)

8. A. Amphawan and S. Chaudhary, International Conference on Optical and Photonic Engineering (icOPEN2015), pp. 95242H-95242H-6: (2015)

9. I. M. Fazal et al., Optics letters, 37, no. 22, pp. 4753-4755 (2012)

10. H. Huang et al., Optical Fiber Communication Conference, p. OTh4G. 5: Optical Society of America (2013)

11. H. Huang et al., Optics letters, 39, no. 2, pp. 197-200 (2014)

12. Y. Ren et al., Optics letters, 38, no. 20, pp. 4062-4065 (2013)

13. A. Amphawan, Optics express, 19, no. 23, pp. 2308523096 (2011)

14. A. Amphawan, V. Mishra, K. Nisar, and B. Nedniyom, Journal of Modern Optics, 59, no. 20, pp. 1745-1752 (2012)

15. S. O. Arik, J. M. Kahn, and K.-P. Ho, Signal Processing Magazine, IEEE, 31, no. 2, pp. 25-34 (2014)

16. A. Amphawan, Optics express, 19, no. 10, pp. 9056-9065 (2011)

17. A. Amphawan, Journal of Modern Optics, 59, no. 5, pp. 460-469 (2012)

18. J. Carpenter and T. D. Wilkinson, Journal of Lightwave Technology, 30, no. 12, pp. 1978-1984 (2012)

19. R. Ryf et al., Optical Fiber Communication Conference, p. PDPB10: Optical Society of America (2011)

20. O. Leclerc et al., Journal of Lightwave Technology, 21, no. 11, p. 2779 (2003)

21. P. J. Winzer and R.-J. Essiambre, Proceedings of the IEEE, 94, no. 5, pp. 952-985 (2006)

22. "Tools of the Trade," 20, Thorlabs, Ed., ed (2010)

23. A. Ghatak and K. Thyagarajan, An introduction to fiber optics (1998)

24. E. J. McCartney, "Optics of the atmosphere: scattering by molecules and particles," 421 p., 1 (1976)

25. I. I. Kim, B. McArthur, and E. Korevaar, Proc. SPIE, 4214, pp. 26-37 (2001)

26. A. K. Majumdar, Free-Space Laser Communications: pp. 57-108 (2008)

27. A. Amphawan and D. O'Brien, IEEE Int. Conf. Photon. (ICP), Langkawi, pp. 1-5: (2010) 